\title{
Automatic Registration of the Nascence and Propagation of Cracks in Electrically Conductive Materials
}

\author{
Vyacheslav M. Kiiko ${ }^{1, a^{*}}$, Kirill A. Khvostunkover,b and Konstantin A. Fedotove,c \\ ${ }^{1}$ Institute of Solid State Physics of the Russian Academy of Sciences, Chernogolovka Moscow \\ district, 142432 Russia \\ ${ }^{2}$ Moscow State University, Moscow, 119991 Russia \\ a kiiko@issp.ac.ru, bkhvostunkov@gmail.com, fedotishn@mail.com
}

Keywords: Electrically Conductive Specimen, Equipotential Lines, Crack, Conformal Mapping, Automatic Fracture Control

\begin{abstract}
A correlation is established between the length of the propagating crack in electrically conductive flat specimens and the change in the field of electric potentials over the surface of the specimen when an electric current is passed through it. The experimental data correspond to the analytical solution in a two-dimensional formulation obtained by means of a conformal mapping. Work can be attributed to the field of converting mechanical values into electrical ones. This transformation simplifies the process of recording mechanical processes and provides a convenient form of their control. The work is aimed at automatic control of the process of fracture of electrically conductive materials.
\end{abstract}

\section{Introduction}

At present, theoretical and experimental methods of fracture control are being actively developed, for example, based on the analysis of changes in the electromagnetic field [1] or lines of equal potential when passing an electric current through the material [2].In principle, such methods of converting mechanical quantities into electrical ones are capable of providing automatic control of the process of mechanical testing of specimens for fracture toughness and control over the occurrence and propagation of cracks in structural elements operating under load.

This work is aimed at calculating and automatic registration of the occurrence and position of a crack in electrically conductive materials or materials with such a coating. The combination of analytical solutions and automatic crack registration is the main difference from the existing techniques in this area [3-6]. The basis of the work is to establish a one-to-one correspondence between the position of the crack and the geometry of the distribution of equipotential lines in the specimen when an electric current is passed through it. The crack is assumed to be rectilinear, inclined at an arbitrary angle to the boundary of the strip (hereinafter referred to as "sample") made of conductive material. The geometry of the equipotential lines does not depend on the electrical resistance of the material and the magnitude of the current passed through it, since these quantities are not included in the Laplace equations describing the positions of the equipotential lines [2]. The technique does not require preliminary experimental calibration of the crack position; it is provided by calculations performed on the basis of analytical solutions by the method of conformal mappings. Experimental modeling was carried out on sheet thermally split graphite, and laboratory tests - on sheet material made of aluminum alloy.

\section{Analytical solution}

Consider a rectangular strip $L \times H$, of electrically conductive material, located with its extended side $L$ along the axis $x$, containing a rectilinear edge crack directed at an angle $\alpha$ to the axis $x$ and 
with length $l, l<<H<<L$. Assume that the crack size $l$ is small compared with the characteristic width of the strip $H$. It is known that the crack increases in its length. It is necessary to determine the dependence of the change in the geometry of equipotential lines during the growth of the crack at a constant value of the potential difference at the ends of the strip (Fig. 1a, Fig. 1b).

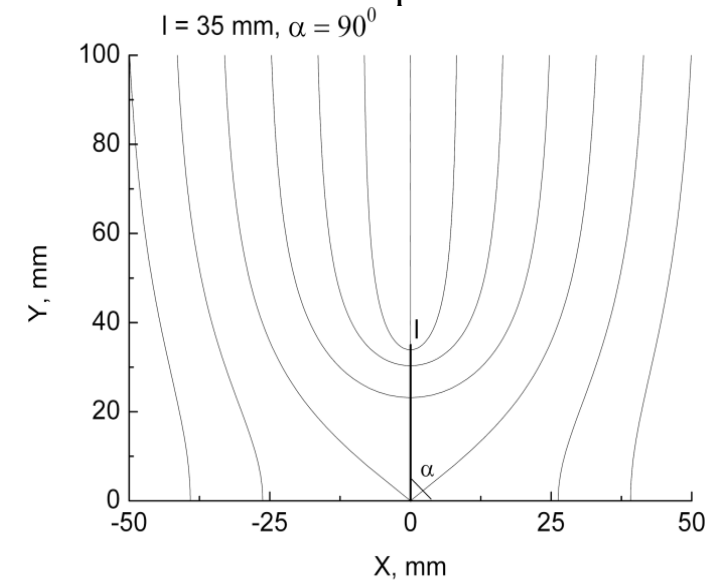

(a)

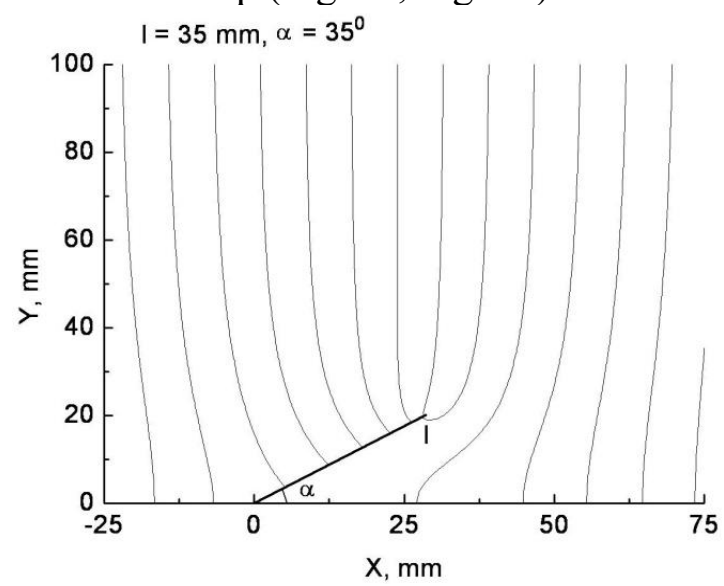

(b)

Fig. 1. (a) - the case of a crack perpendicular, $\alpha=90^{\circ}$, to the sample boundary, (b) - the case of an arbitrary inclination $\alpha$ of the crack to the boundary.

Due the $l<<H$, we will assume that the equipotential lines at the upper boundary of the band are parallel and we will further consider the half-plane. As in the case of the perpendicular crack in Fig. 1a [2], we will use the conformal mapping method.

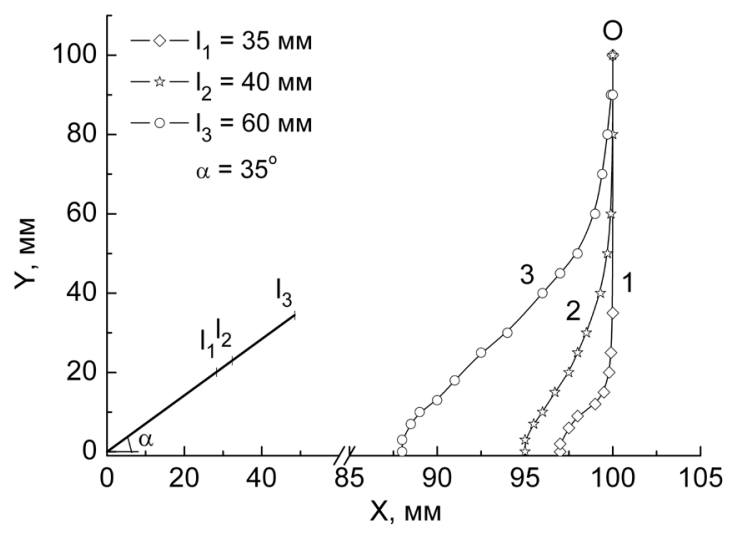

(a)

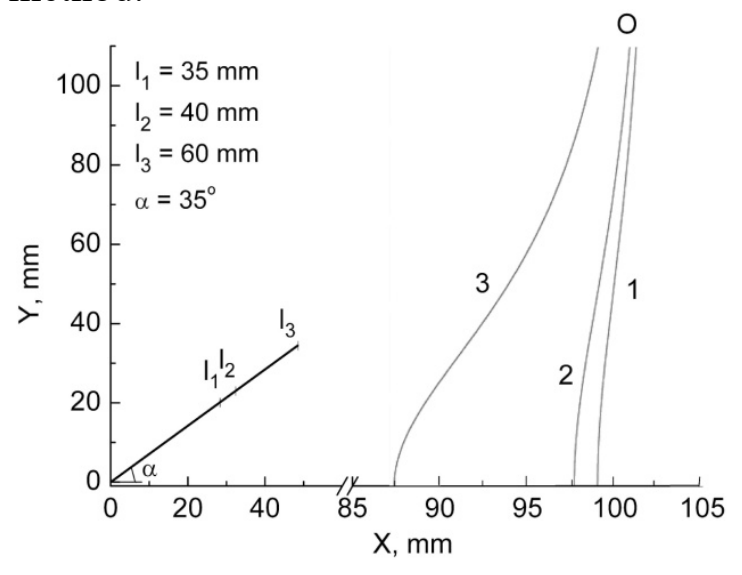

Fig. 2. (a) - experimentally obtained equipotential lines passing through point $O$ (100, 100), at different lengths $l$ of an oblique crack in a specimen of sheet thermally split graphite 100 $\mathrm{mm}$ vertically wide, $320 \mathrm{~mm}$ horizontally long and $1.5 \mathrm{~mm}$ thick; (b) - calculated lines, $O$ $(100, \infty)$. 
The conformal mapping, which translates the half-plane into our desired half-plane with the crack, has the form [7,8]: $w=l(z-1)^{\frac{\alpha}{\pi}}\left(1-\frac{\alpha z}{\pi-\alpha}\right)^{1-\frac{\alpha}{\pi}}, w=x+i y, \quad z=\xi+i \eta$. It is known that the solution of the Laplace equation for equipotential lines is invariant under conformal mapping. Fig. $2 \mathrm{a}$ and Fig. $2 \mathrm{~b}$ present examples of the behavior of the fixed line as the crack length increases.

The theoretical part of the work consists in determining the velocity of the point $\mathrm{N}$ along the axis $x$ at the level of the upper edge of the sample (Fig. 3b, Fig. 4a) belonging to the equipotential line passing through a predetermined fixed point M, (Fig. 4a). For this purpose, let us define the real and imaginary parts of mapping (Eq. 1):

$$
\begin{aligned}
& x(\xi, \eta)=l \rho_{1}^{\frac{\alpha}{\pi}} \rho_{2}^{1-\frac{\alpha}{\pi}} \cos \left(\frac{\alpha}{\pi} \theta_{1}+\left(1-\frac{\alpha}{\pi}\right) \theta_{2}\right), \quad y(\xi, \eta)=l \rho_{1}{ }^{\frac{\alpha}{\pi}} \rho_{2}{ }^{1-\frac{\alpha}{\pi}} \sin \left(\frac{\alpha}{\pi} \theta_{1}+\left(1-\frac{\alpha}{\pi}\right) \theta_{2}\right) \\
& \rho_{1}=\sqrt{(\xi-1)^{2}+\eta^{2}}, \theta_{1}=\operatorname{arctg}\left(\frac{\eta}{\xi-1}\right), \rho_{2}=\frac{\alpha}{\pi-\alpha} \sqrt{\left(\frac{\pi}{\alpha}-1-\xi\right)^{2}+\eta^{2}}, \theta_{2}=\operatorname{arctg}\left(\frac{\eta}{\frac{\pi}{\alpha}-1-\xi}\right) .
\end{aligned}
$$

Each equipotential line is parameterized by $\eta$ as a one-parameter curve with an individual number $\xi=$ Const . Then it is necessary to determine which curve passes through point $\mathrm{M}$ for a given crack length $l$, and which coordinate $x$ it will have at the level of $y=H$. From the condition that the equipotential line passes through both points $\mathrm{M}$ and $\mathrm{N}$, we find the position along $\mathrm{x}$ at the level of $y=H$.

$$
\begin{aligned}
& \operatorname{tg}\left(\frac{\alpha}{\pi} \operatorname{arctg}\left(\frac{\eta}{\xi-1}\right)+\left(1-\frac{\alpha}{\pi}\right) \operatorname{arctg}\left(\frac{\eta}{\frac{\pi}{\alpha}-1-\xi}\right)\right)=A, \quad A=\frac{y_{M}}{x_{M}} \\
& \left(\frac{l}{x_{M}}\right)^{2}\left(\sqrt{(\xi-1)^{2}+\eta^{2}}\right)^{2 \frac{\alpha}{\pi}}\left(\frac{\alpha}{\pi-\alpha} \sqrt{\left(\frac{\pi}{\alpha}-1-\xi\right)^{2}+\eta^{2}}\right)^{2-2 \frac{\alpha}{\pi}}=1+A^{2}
\end{aligned}
$$

In order to solve the system (Eq. 2), let us assume specific values for the positions of control points at the outer edge and at a distance $H$ from it, $\eta_{M}=0, \quad \eta_{H}=1, \quad A=0, \quad a=\frac{\pi}{\alpha}-1$

$$
\left(\frac{l}{x_{m}}\right)^{a+1}=\frac{1}{(\xi-1)\left(1-\frac{\xi}{a}\right)^{a}}, \quad\left(\frac{x}{x_{m}}\right)^{2}=\left(\frac{l}{x_{m}}\right)^{2}\left((\xi-1)^{2}+1\right)^{\frac{1}{a+1}}\left(\left(1-\frac{\xi}{a}\right)^{2}+\left(\frac{1}{a}\right)^{2}\right)^{\frac{a}{a+1}}-\left(\frac{H}{x_{m}}\right)^{2}
$$

On the interval of strict monotonicity at $\xi \in\left(1, \frac{2 a}{a+1}\right)$ the dependence $\left(l / x_{m}\right)^{a+1}=(\xi-1)^{-1}\left(1-\frac{\xi}{a}\right)^{-a}$ is reversible and we can express the number of the equipotential line 
passing through the $x$-axis as a function of the dimensionless crack length: $\xi=f\left(l / x_{m}\right)$. In each special case, the function $f$ can be obtained numerically, and in the case of $a=2$, (it corresponds to a crack slope angle of 60 degrees), it can be calculated analytically by the Ferrary-Cardano method.

As a result, we obtain the dependence of the coordinate of the second end of this equipotential line at distance $H$ from the bottom edge:

$$
\frac{x}{x_{m}}=\frac{l}{x_{m}}\left\{\left[\left(f\left(\frac{l}{x_{m}}\right)-1\right)^{2}+1\right]^{\frac{1}{a+1}}\left[\left(1-\frac{1}{a} f\left(\frac{l}{x_{m}}\right)\right)^{2}+\left(\frac{1}{a}\right)^{2}\right]^{\frac{a}{a+1}}-\left(\frac{H}{l}\right)^{2}\right\}^{\frac{1}{2}} .
$$

\section{Experiments}

As already mentioned, the distribution of electric potentials in the specimen is established by an analytical solution based on the use of a conformal mapping in a two-dimensional formulation, and the calculation results are compared with the experimental data (Fig. 2, Fig. 3). This opens up the possibility of establishing a one-to-one correspondence of the geometry of the developing crack with the geometry of the distribution of electric potentials and automatic control of experiments related to the propagation of a crack in the material of the specimen [2], and, in the future, in real structure elements.

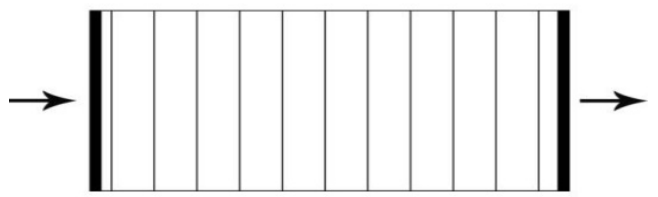

(a)

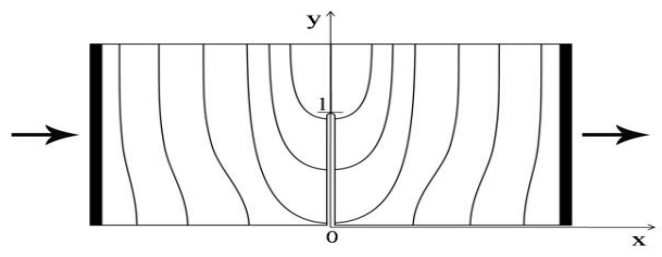

(b)

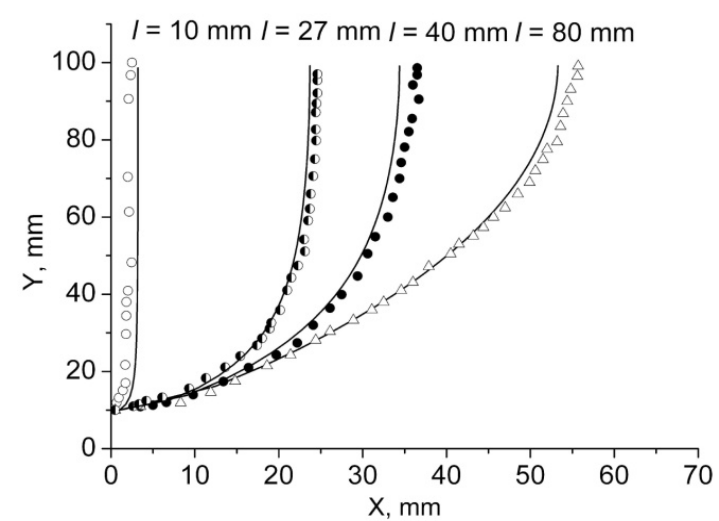

(c)

Fig. 3. Scheme of the arrangement of equipotential lines over the surface of a flat specimen when passing through it an electric current, shown by arrows: (a) - a specimen without a crack, (b) - with a lateral crack of length l, perpendicular to the lower edge of the specimen; (c) - the position of the equipotential lines passing through the point (0.10) - in millimeters, depending on the lengths of the lateral crack (indicated in the graph field) with the specimen width (along the height of the figure) 100 millimeters: solid lines - the results of the analytical solution, "points" - determination of the position of equipotential lines experimentally.

The scheme of tracking the change in the coordinate $\mathrm{x}$ of the "exit" of the equipotential line passing through the fixed point of contact $\mathrm{M}$ located at the beginning of the crack to the upper edge of the sample is shown in Fig. 4a. A fixed measuring contact is placed at point M. By moving the movable measuring contact along the upper edge of the specimen, its position is found at which the voltage between the points $\mathrm{M}$ and $\mathrm{N}\left(\mathrm{x}_{1}\right)$ is zero, which is controlled by the readings of the 
voltmeter $\mathrm{V}$. In this case, points $\mathrm{M}$ and $\mathrm{N}$ will be on the same equipotential line. An increase in the crack length from $l_{1}$ to $l_{2}$ by $\Delta 1$ leads to a change in the geometry of the potential field and, therefore, the equipotential line passing through the point $\mathrm{M}$ will take the position $\mathrm{MN}^{\prime}$. On the edge there will be a new point $\mathrm{N}^{\prime}$, different in position from point $\mathrm{N}$, such that there will be no voltage between $\mathrm{M}$ and $\mathrm{N}^{\prime}$. The new position $\mathrm{N}^{\prime}\left(\mathrm{x}_{2}\right)$ of the movable contact is found by moving the contact from point $\mathrm{N}$ to $\Delta \mathrm{x}$ along the edge of the specimen. Thus, a one-to-one correspondence is established between the increment in the crack length $\Delta \mathrm{l}=1_{2}-1_{1}$ and the displacement of the contact $\Delta \mathrm{x}=\mathrm{x}_{2}-\mathrm{x}_{1}$. Moving a contact can be done either manually or automatically. In Fig. $4 \mathrm{~b}$ shows the results obtained manually, automatically, and by calculation.

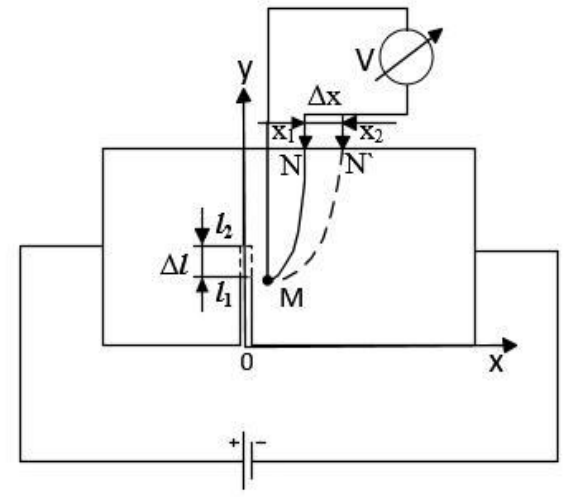

(a)

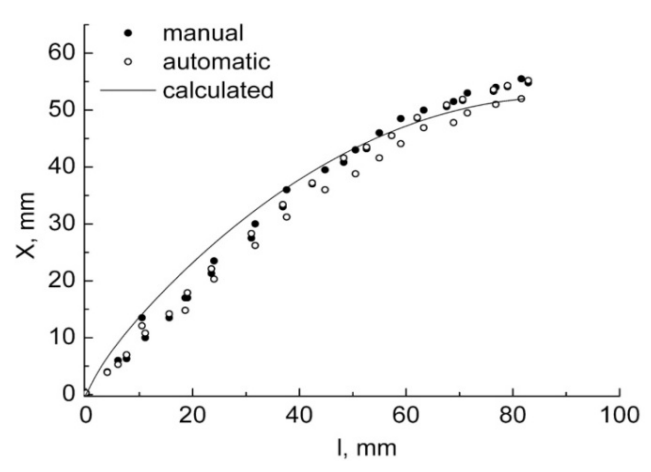

(b)

Fig. 4. Scheme for converting the value of the growth of the crack length $\Delta l$ into the movement of the movable contact $\Delta x(a) ;(b)$-dependences of the displacement $x$ of the movable measuring contact on the length of the lateral crack l, obtained manually, automatically and by calculation.

The dependencies shown in Fig. $4 \mathrm{~b}$ are, in essence, calibration.

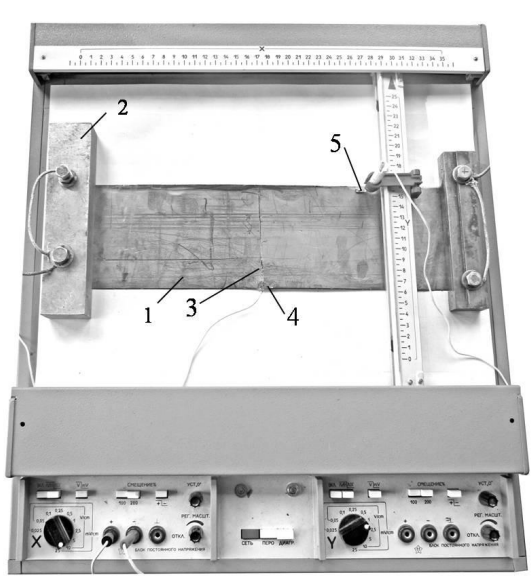

(a)

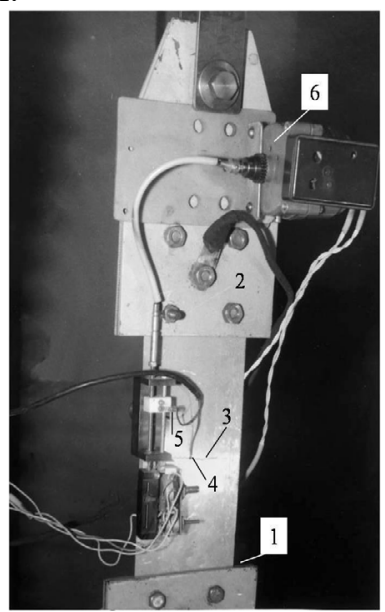

(b)

Fig. 5. A device for carrying out model experiments related to determining the distribution of electrical potentials over the surface of the specimen (a); (b) - electro-mechanical part of a laboratory device for automatic recording of the crack length. 1 -specimen, 2 -current leads, 3

- crack, 4-fixed contact, 5 - movable contact, 6 - reversible electric motor. 
The experimental obtaining of "points" on the graph (Fig. 4b) was carried out in a model version on a strip of sheet thermally split graphite. A direct current was passed through the sample using current leads 2 (Fig. 5a) providing a uniform current distribution over the ends of the specimen. The lateral crack was simulated by cut 3."Manually" the position of the movable contact was determined with a caliper, and the corresponding points are plotted on the graph in Fig. 4b. The automatic device for experiments is based on an industrial two-coordinate recorder H307, adapted for the tasks under consideration. Fixed contact 4 was fixed at the beginning of the crack, through which the initial line of equal potential passed. The other "end" of this line went out to the upper (according to Fig. $1-4 a$ ) edge of the specimen. Its position was also determined automatically. The voltage supplied from the fixed - movable contacts to the input "x" of the recorder controlled the movement of the recorder carriage with the movable contact fixed on it and brought it by the modified recorder circuit to the equipotential line passing through the fixed contact, every time the crack length changed. A specially added multi-start potentiometer, connected with the carriage movement mechanism, included in the bridge electrical circuit, made it possible to obtain at the output of the system electrical voltages corresponding to the position of the carriage. These voltages are a function of the crack length. Carriage positions in the form of "points" are shown in Fig. 4b ("automatic" measurements).

Similar to the model version, (Fig. 5a) a laboratory device was manufactured (Fig. 5b) for automatic measurement of the crack length in sheet specimens made of conductive materials or with such a coating. Tests with automatic registration of the fatigue crack length in a specimen of aluminum alloy D16 are shown in Fig. 5b.

\section{Summary}

In this work, solutions of the tasks of the distribution of equipotential lines in flat specimens by passing an electric current through them are obtained in a two-dimensional formulation. Analytical solutions are based on conformal mappings for specimens with different orientations of edge cracks.

The distributions of equipotential lines were also obtained experimentally on similar specimens of electrically conductive thermally split graphite on a specially made simulator.

The numerical results obtained on the basis of analytical solutions and the results obtained experimentally coincide with the accuracy acceptable for practice. These coincident results make it possible to abandon the procedure for calibrating specimens before testing.

Developed, manufactured and used one of the possible electronic-mechanical device for automatic registration of the crack length in conductive materials or with such a coating. Laboratory tests with a developing fatigue crack were carried out on sheet D16 aluminum alloy.

\section{Acknowledgments}

The work was carried out within the framework of State Assignment No. 0028-2019-0020. Also the authors are grateful to R K Odzhaev and D G Pizhenin for help in computational and experimental work.

\section{References}

[1] Ni Qing-Qing, J. Hong, P. Xu, Xu Zhenzhen, K.A. Khvostunkov, H. Xia, Damage detection of CFRP composites by electromagnetic wave nondestructive testing (EMW-NDT), J.Composites Science and Technology. 211 (2021) 108839. https://doi.org/10.1016/j.compscitech.2021.108839 
[2] V.M. Kiiko, K.A. Khvostunkov, R.K. Odzhaev, D.G. Pizhenin, S.A. Abashkin, Automatic registration of the length of the propagating crack in conductive materials, in V.V. Rubanik (Ed.), Actual problems of strength, Publishing "Pobeda", Molodechno, 2020, pp. 251-253. (in Russian).

[3] F.R. Sosnin, K.V.Podmasterev,Unbrakable control, Moscow, 2004. (in Russian)

[4] P.N. Shkatov, P.I. Chernenko, Measurement of the depth and angle of inclination of surface cracks using the electropotentialmethod, Vestnik MGUPI, 44 (2013). (in Russian)

[5] L.N. Litvinov, F.G.Tsipushtanov, V.A. Parkov, V.N. Bakumov,Electropotential Crack Depth Gauge, Patent RF, 1408205 (1988). (in Russian)

[6] D.A.Ozetskiy, V.V. Tsvelev, Method for measuring crack propagation in conductive specimens, Patent RF, 1834491 (1966). (in Russian)

[7] M.A. Lavrentiev, B.V. Shabat, Methods of the theory of functions of a complex variable, Moscow, 2002. (in Russian)

[8] Y.V. Sidorov, M.V. Fedoryuk, M.I. Shabunin, Lectures on the theory of functions of a complex variable, Moscow, 1989. (in Russian) 